\title{
Steroid-induced Spinal Epidural Lipomatosis after Suprasella Tumor Resection
}

\author{
Si On Kim ${ }^{1,2}$, Keuk Kyu Park ${ }^{1}$, Young Jun Kwon ${ }^{1}$, Hyun Chul Shin ${ }^{1}$, Chun Sik Choi ${ }^{1}$ \\ ${ }^{l}$ Departments of Neurosurgery, Kangbuk Samsung Hospital, Sungkyunkwan University College of Medicine, Seoul, \\ ${ }^{2}$ Department of Medicine, The Graduate School, Yonsei University, Seoul, Republic of Korea
}

Spinal epidural lipomatosis (SEL) is an abnormal localized accumulation of fat tissues in the epidural space. It is strongly related with steroid administration. The symptoms of SEL are various and range from back pain to paraplegia. In severe cases, decompressive laminectomy is the choice of treatment. A 32-year-old woman who had been under long-term steroid administration after suprasellar tumor resection was admitted for both leg radiating pain and weakness. She was diagnosed with SEL and had a decompressive laminectomy. During the operation, we found the nerve roots were compressed by epidural fat tissues and engorged vessels. After the operation, her radiating pain was relieved and motor weakness was improved.

Key Words: Spinal epidural lipomatosis · Suprasellar tumor $\cdot$ Steroid

\section{INTRODUCTION}

Spinal epidural lipomatosis (SEL) is a rare disease that is characterized by pathologic overgrowth of lumbar or thoracic epidural fat tissue. Although the etiology of SEL is not known, SEL has been frequently diagnosed in obese patient groups and exogenous steroid administration groups, so steroid metabolism is presumed to be related with SEL. Excessive epidural fat deposition in the spinal canal can contribute to various neurologic symptoms, such as back pain, radiculopathy, myelopathy, claudication and even cauda equina syndrome. In this case, we present a SEL patient who had taken steroid hormone medicine for 20 years, after a suprasellar tumor resection.

\section{CASE REPORT}

A 32-year-old woman was admitted in a wheelchair. She presented with leg weakness and radiating pain on both buttock, posterior thigh \& calf for 1 year. Because pain and weak-

- Received: February 2, 2013 - Revised: May 3, 2013

- Accepted: May 8, 2013

Corresponding Author: Chun Sik Choi, MD

Departments of Neurosurgery, Kangbuk Samsung Hospital, Sungkyunkwan

University College of Medicine, Pyung-dong 108, Jongro-gu, Seoul 110-746,

Republic of Korea

Tel: +82-2-2001-2158, Fax: +82-2-2001-2157

E-mail: cs8.choi@samsung.com ness in both legs was gradually aggravated during 4 months, she could not walk or stand when she was admitted. Prior to admission, she underwent an operation for a suprasellar tumor resection in our hospital when she was 14 years old. At that time, the pathologic diagnosis was pilocytic astrocytoma. After the operation, panhypopituitarism occurred, so steroid hormone replacement therapy was started in 1993. For a period of 19 years, exogenous steroid maintenance had been administrated (median dose of prednisolon $7.5 \mathrm{mg}$ ). The neurologic examination revealed both legs weakness (motor grade IV/IV) and radiating pain on the L5 and S1 dermatomes. The straight leg raising test was negative, but the bilateral knee jerk and ankle jerk were slightly hyperreflexive. A magnetic resonance imaging (MRI) study showed severe compression of the lumbar spinal cord by epidural fat tissue. At the dorsum of the spinal cord from the L1 to L5 levels, high signal lesions on T1- and T2-weighted images were checked. The longest length from the dorsum of the spinal cord to the ligamentum flavum was $11.4 \mathrm{~mm}$ on the $\mathrm{L} 3$ level and the median length was $9.2 \mathrm{~mm}$ at the L1-L5 levels. Fat suppression MR imaging showed low signal intensity at the same lesion (Fig. 1) as well. In the enhanced T1-weighted MRI, the epidural vessels were more developed than in a normal person's MRI. The engorged epidural vessels were positioned beneath the nerve root exit site, bilaterally (Fig. 2).

Considering the long-term exogenous steroid administration, her neurologic symptoms and MR images, we could make the diagnosis of spinal epidural lipomatosis. Because she complained of radiating pain on the bilateral L5 and S1 derma- 

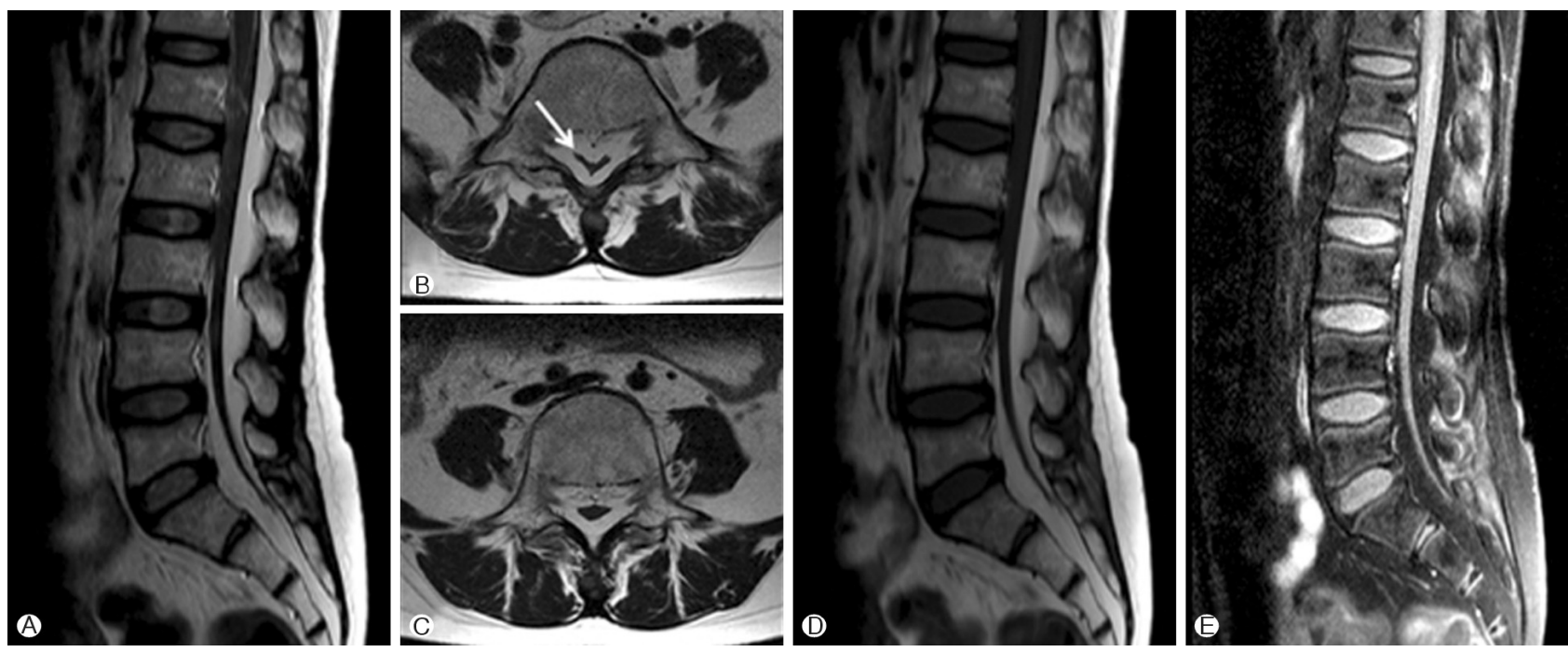

Fig. 1. A, B and C: Preoperative sagittal, axial T2-weighted MRI. The thecal sac is compressed by epidural fat tissue on the L1-5 levels. In the axial T2-weighted MRI, nerve roots are concentrated and CSF in the thecal sac is not observed by epidural fat compression. The white arrow is the compressed thecal sac. This thecal sac resembled the letter "Y" (Y-sign) $)^{5)} \mathrm{D}$ : Preoperative sagittal T1weighted MRI, E: Preoperative sagittal fat suppression MRI.
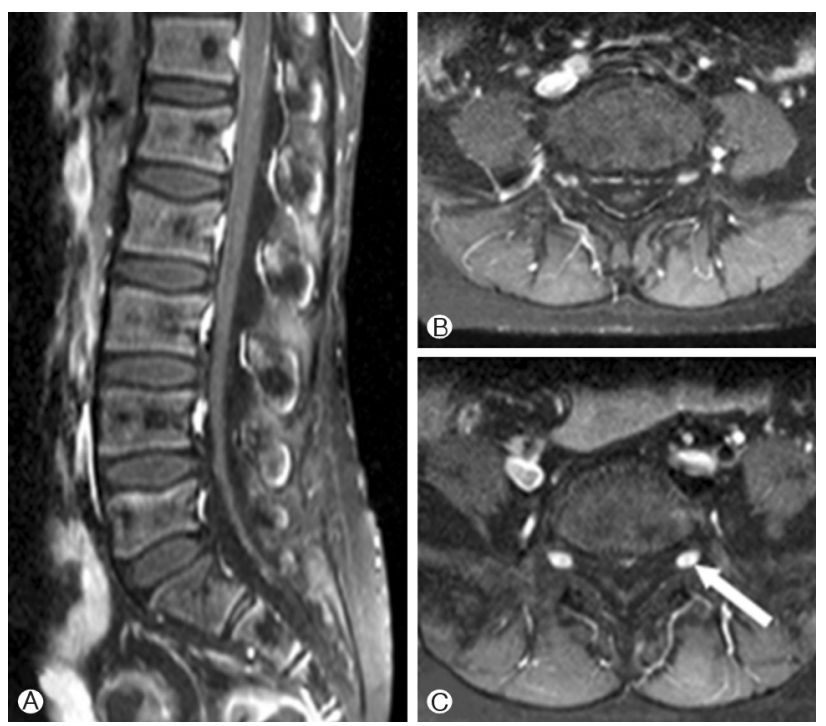

Fig. 2. A, B and C: Preoperative sagittal, axial enhanced T1weighted MRI. The white arrow is an engorged epidural vessel which is compressing the $\mathrm{L} 5$ nerve roots. The median diameter of the epidural vessels is $5.6 \mathrm{~mm}$. They are very enlarged, enough to lead to radiculopathy.

tomes, she had an operation to decompress the L5 and S1 nerve roots. In the operation field, fat tissue compressed the dorsum of the thecal sac and engorged epidural vessels were found on nerve root exit sites (Fig. 3). We removed the epidural fat tissue and coagulated the engorged epidural vessels for nerve root decompression. The postoperative course was good. The radiating pain in both legs was dramatically relie-
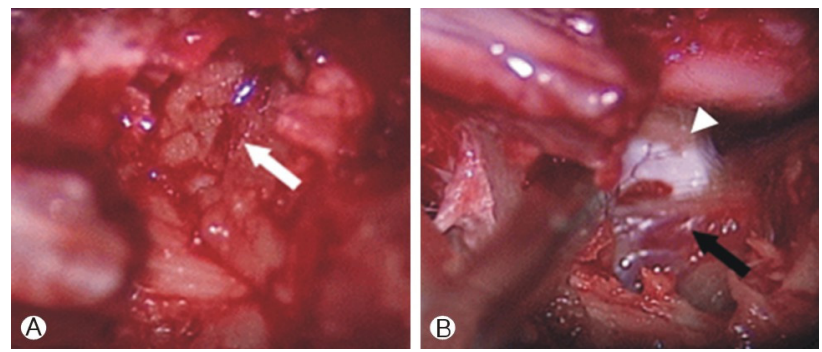

Fig. 3. A: The white arrow is epidural fat tissue, which is covering the dorsal aspect of the $L 5$ nerve root, B: The white arrow head is the $L 5$ nerve root. The black arrow is the engorged epidural vessel, which is compressing the $L 5$ nerve root.

ved and motor power was slightly improved. She was admitted in a wheelchair but discharged walking. After 1 month, we did a follow-up MRI. On the follow-up MRI, the epidural fat tissue did not compress the thecal sac and the courses of nerve roots were not interrupted.

\section{DISCUSSION}

Spinal epidural lipomatosisis a rare disease characterized by overgrowth of epidural fat tissue, causing compression of the spinal neural components. SEL was first reported by Lee et al. ${ }^{11)}$ in 1975 . The first reported patient was using steroids after a renal transplantation. After the first report, subsequent reports about this disease have been published, but the pathological mechanism of SEL still remains unclear ${ }^{16}$. SEL is frequently associated with the administration of exogenous ste- 
roids and obesity. There are many case reports about SEL patients who were under long-term steroid therapy for curing ulcerative colitis, asthma, sarcoidosis, rheumatoid arthritis or renal disease ${ }^{3,8,10,12,15)}$. But in some cases, it is not related to steroids and obesity.

Al-Khawaja et al. ${ }^{2)}$ reported that SEL is classified in the idiopathic group and secondary group. In this review, they studied 111 patients, and both idiopathic and secondary groups are strongly male-predominant. In the idiopathic group, lumbar segments are more involved than thoracic segments and leg pain and back pain are the most frequent symptoms. Relatively, in the secondary group (steroid administration or endocrinopathy group), thoracic segments are more involved than lumbar segments and myelopathy is the main symptom.

The symptoms of SEL are dependent on the level of spinal canal compromise ${ }^{2,4)}$. In reported cases, the symptoms are gradual onset and similar to degenerative spinal stenosis. And the severity of symptoms are various from painful back pain to paraplegia. MRI is the best diagnostic test for evaluating SEL. The epidural fat tissue can be easily measured on T1weighted MR images ${ }^{17)}$. The diagnostic criterion of SEL is greater than $6 \mathrm{~mm}$ thickness of epidural fat tissue. Possible differential diagnoses include encapsulated spinal lipomas, spinal stenosis, and degenerative joint disease, which can also cause spinal compression and present with similar symptoms ${ }^{17}$.

The treatments of SEL depend on the severity of neurologic manifestations ${ }^{14)}$. Conservative managements such as exogenous steroid tapering and weight loss are recommended in early SEL patients while patients with severe symptoms or progressive neurologic deficits are indicated for operation ${ }^{1,7,9,13)}$. In a study by Fogel et al. ${ }^{7}$, the results of surgery were good. Seventy-seven percent of patients of the steroid group who underwent on decompressive laminectomy had symptom improvements. And the success rate of the obese group was 66.7\%. But in a study by Fassett et al. ${ }^{\circ}$, the mortality rate in these patients within 1 year after surgical decompression was $22 \%$, because of concomitant medical problems and comorbidities. Consequentially, we must make a treatment plan that considers various factors, such as neurologic severity, general health, steroid therapy and lifestyle.

\section{CONCLUSION}

In patients who underwent an operation for suprasellar lesions such as pituitary adenoma, suprasellar meningioma, craniopharyngioma, and so forth, we can occasionally observe complications from corticosteroid hormone deficiency; in which case, we start corticosteroid hormone replacement therapy without worrying about the SEL. In our case, we reviewed the studies showing that long-term corticosteroid replacement therapy after neurosurgery can lead to spinal epidural lipomatosis

\section{REFERENCES}

1. Akhaddar A, Ennouali H, Gazzaz M, Naama O, Elmostarchid B, Boucetta M: Idiopathic spinal epidural lipomatosis without obesity: a case with relapsing and remitting course. Spinal Cord 46:243-244, 2008

2. Al-Khawaja D, Seex K, Eslick GD: Spinal epidural lipomatosis - A brief review. J Clin Neurosci 15:1323-1326, 2008

3. Arroyo IL, Barron KS, Brewer EJ Jr: Spinal cord compression by epidural lipomatosis in juvenile rheumatoid arthritis. Arthritis Rheum 31:447-451, 1988

4. Chan JY, Chang CJ, Jeng CM, Huang SH, Liu YK, Huang JS: Idiopathic spinal epidural lipomatosis - two cases report and review of literature. Chang Gung Med J 32:662-667, 2009

5. De Cocker L Jr, De Cocker L Sr, Van Rillaer O, Ceuppens J, van Loon J: The Y-sign in spinal epidural lipomatosis. JBR-BTR 92:235, 2009

6. Fassett DR, Schmidt MH.: Spinal epidural lipomatosis: a review of its causes and recommendations for treatment. Neurosurg focus 15:16, 2004

7. Fogel GR, Cunningham PY 3rd, Esses SI: Spinal epidural lipomatosis: case reports, literature review and meta-analysis. Spine J 5:202-211, 2005

8. Kano K, Kyo K, Ito S, Nishikura K, Ando T, Yamada Y, et al.: Spinal epidural lipomatosis in children with renal diseases receiving steroid therapy. Pediatr Nephrol 20:184-189, 2005

9. Kawai M, Udaka F, Nishioka K, Houshimaru M, Koyama T, Kameyama M: A case of idiopathic spinal epidural lipomatosis presented with radicular pain caused by compression with enlarged veins surrounding nerve roots. Acta Neurol Scand 105:322325,2002

10. Kotilainen E, Hohenthal U, Karhu J, Kotilainen P: Spinal epidural lipomatosis caused by corticosteroid treatment in ulcerative colitis. Eur J Intern Med 17:138-140, 2006

11. Lee M, Lekias J, Gubbay SS, Hurst PE: Spinal cord compression by extradural fat after renal transplantation. Med J Aust 1:201203, 1975

12. Montoriol PF, Da Ines D, Bailly A, Garcier JM: Steroid-induced epidural lipomatosis in a patient with sarcoidosis. J Radiol 91: 511-513, 2010

13. Park SH, Roh SW, Sung KH: Idiopathic Spinal Epidural Lipomatosis: A Case Report. Korean J Spine 2:65-67, 2005

14. Venkatanarasimha N, Parrish RW: Thoracic epidural lipomatosis. Radiology 252:618-622, 2009

15. Wakerley B, Kent L, Jackson M: Progressive epidural lipomatosis with steroid use in severe refractory asthma. J Asthma 48: 316-318, 2011

16. Wälchli B, Benini A: Spinal epidural lipomatosis. Swiss Med Wkly 131:359-360, 2001

17. Zhang Y, Wood MJ, Gilligan C: Spinal cord stimulation in a patient with spinal epidural lipomatosis. Pain Med 12:377-81, 2011 